\title{
Anabases
}

ANABASES Traditions et réceptions de l'Antiquité

6 | 2007

Varia

Fiona MACINTOSH, Pantelis MICHELAKIS, Edith HALL \& Oliver TAPLIN (ed.), Agamemnon in Performance. 458 BC to AD 2004

\section{Marie-Hélène Garelli}

\section{OpenEdition}

Journals

Édition électronique

URL : http://journals.openedition.org/anabases/3398

DOI : $10.4000 /$ anabases.3398

ISSN : 2256-9421

Éditeur

E.R.A.S.M.E.

Édition imprimée

Date de publication : 1 octobre 2007

Pagination : 255-258

ISSN : 1774-4296

Référence électronique

Marie-Hélène Garelli, «Fiona macintosh, Pantelis michelakis, Edith hall \& Oliver taplin (ed.), Agamemnon in Performance. 458 BC to AD 2004 », Anabases [En ligne], 6 | 2007, mis en ligne le 01 janvier 2012, consulté le 21 septembre 2020. URL : http://journals.openedition.org/anabases/3398 ; DOI : https:// doi.org/10.4000/anabases.3398

Ce document a été généré automatiquement le 21 septembre 2020

(c) Anabases 


\title{
Fiona MACINTOSH, Pantelis MICHELAKIS, Edith HALL \& Oliver TAPLIN (ed.), Agamemnon in Performance. 458 BC to AD 2004
}

\author{
Marie-Hélène Garelli
}

\section{RÉFÉRENCE}

Fiona Macintosh, Pantelis Michelakis, Edith Hall \& Oliver Taplin (ed.), Agamemnon in Performance. 458 BC to AD 2004, Oxford University Press, 2005, 484 p.

110 euros/ isbn 1-19-926351-5.

1 Cinq ans après Medea in Performance (publié en 2000, sous la direction conjointe d'Edith Hall et Oliver Taplin, par l'European Humanities Research Centre), ce nouveau volume, Agamemnon in Performance, composé dans le même esprit, sous la direction savante de membres éminents de l'apgrd, Edith Hall et Oliver Taplin, Fiona Macintosh et Pantelis Michelakis, témoigne de la vitalité de la recherche anglo-saxonne sur la réception de l'Antiquité, notamment celle du théâtre antique. L'apgrd (Archive of Performances of Greek and Roman Drama, Centre de l'Université d'Oxford), collecte, traite et étudie les documents relatifs aux représentations de pièces antiques depuis la Renaissance jusqu'à nos jours. Ce travail considérable de collecte et d'archivage a produit, entre autres, une base de données (accessible sur www.apgrd.ox.ac.uk), outil d'une richesse extraordinaire et d'une indéniable utilité pour les chercheurs du monde entier. Les travaux du Centre aboutissent également à des publications collectives régulières. La série compte déjà plusieurs ouvrages publiés, pour la plupart, aux Oxford University Press, à partir des recherches effectuées dans le cadre de l'apgrd: Greek Tragedy and the British Theatre 1660-1914, par E. Hall et F. Macintosh (2005); Dionysus since 69, Greek Tragedy at the Dawn of the Third Millenium, par E. Hall, F. Macinthosh et A. Wrigley (2004). 
2 L'ouvrage s'adresse principalement à un public de chercheurs. Il est le fruit d'une collaboration entre universitaires de tous horizons, géographiques et disciplinaires (deux tiers des contributions sont le fait d'antiquisants, les autres sont signées par des spécialistes des arts du spectacle, de littérature comparée, de littérature anglaise). Les problématiques transversales sont posées avec clarté par Pantelis Michelakis, dans une introduction qui synthétise l'apport des contributions tout en définissant les grandes orientations scientifiques. L'unité de la trilogie est d'emblée mise en question: la lecture de l'Orestie comme un ensemble unitaire et cohérent (Agamemnon-ChoéphoresEuménides) est une lecture moderne. Avant le xix siècle, Agamemnon était lu et transmis de façon indépendante. Une approche historique, littéraire et iconographique de la question (articles de P. Easterling et E. Hall) nuance considérablement cette conception « unitaire» largement répandue aujourd'hui. À propos de l'Agamemnon, réception antique et réception moderne doivent faire l'objet d'enquêtes séparées, qui supposent une méthodologie, une approche historique et conceptuelle différentes. La grandeur («bigness») de l'Agamemnon d'Eschyle ne fut pas aussi prisée des Anciens (voir la critique d'Aristophane) que de Goethe, Wagner ou des poètes romantiques anglais.

Les différentes contributions sont réparties en quatre rubriques. Ces principes de base justifient l'organisation de l'ouvrage: les deux premières rubriques opposent la réception antique (les sources) et la réception moderne. Le théâtre latin d'époque républicaine transmet l'image d'une Clytemnestre amoureuse, jalouse, angoissée aussi, celle même que l'on retrouve chez Ovide (Art d'aimer) et chez Sénèque (Agamemnon), délaissant le modèle de l'être androgyne et autoritaire, et l'interprétation politique proposée par Eschyle. C'est le modèle forgé par Ovide et Sénèque qui a influencé le plus fortement les différents Agamemnon de la Renaissance. Bien différente est la réception moderne, celle des Romantiques (à propos de la prétendue « redécouverte » d'Eschyle par les Romantiques on lira, précisément, les articles de M. Ewans sur la réception d 'Agamemnon en Allemagne et de F. Macintosh sur la réception en Grande-Bretagne), de Virginia Woolf et sa lecture émotionnelle du grec (le fameux "not knowing Greek » finement commenté par Y. Prins), ou de la scène lyrique de Gluck à Darius Milhaud (contribution, notamment, de M. Reynolds). La réception moderne de l'Agamemnon est habitée par les notions de progrès, de raison, de rationalité : ainsi Egisthe devient-il le paradigme du révolutionnaire français sous la Terreur. La mort d'Agamemnon s'est chargée, dans un Occident à la recherche de son identité, d'interprétations politiques, anthropologiques, psychanalytiques, féministes.

4 La traduction fait l'objet d'un groupement spécifique de contributions au sein d'une troisième rubrique. Sur un ton volontairement provocateur, Oliver Taplin montre la puissance d'une traduction controversée, celle d'Harrison, mise en scène par Peter Hall qui, selon lui, recherche l'esprit plus que la lettre, par le biais d'équivalences hardies, rythmiques et imagées. Plus largement, comme les autres contributions de cette série (noms), la question est posée de la fidélité et de la précision de la traduction, qui conduit à délaisser la musique (au sens large de » musicalité ») du texte. Les articles de J.M. Walton (Translation and Transsubstantiation) et de L. Hardwick (Staging Agamemnon : the Language of Translation) mettent en évidence, à partir de diverses expériences du $\mathrm{xx}^{\mathrm{e}}$ siècle (dont certaines sont très récentes comme celle de David Stuttard et de Katie Mitchell en 1999), le lien fondamental qui existe entre représentation et traduction poétique. Lorsque la traduction est destinée à une transposition cinématographique et 
que l'auteur est Pasolini (l'Orestie de 1960), nous entrons dans un univers visuel et musical, où le pouvoir du logos est systématiquement objet de défiance, comme le montre M. Fusillo (Pasolini's Agamemnon).

5 Un quatrième et dernier ensemble passe en revue les Agamemnon modernes sur la scène internationale, qu'il s'agisse d'arts du spectacle et d'arts visuels (théâtre, opéra, cinéma) ou de littérature moderne. Le concept de "nation» est au centre du questionnement. Le festival de Syracuse (Istituto Nazionale del Dramma Antico), aujourd'hui célèbre dans toute l'Europe, fut inauguré en 1914 par une représentation de l'Agamemnon: la représentation de tragédies antiques relevait de la revendication d'un héritage national qui rendait à la Sicile son pouvoir d'attraction culturelle. Les choix contemporains de mise en scène du chœur, révélateurs du traitement de la notion de polis, (article d'A. Bierl), les relectures scéniques de la pièce en Russie (D. Trubotchkin), aux États-Unis (H.P.Foley) ouvrent des voies de réflexion stimulantes sur les relectures politiques contemporaines de l'Agamemnon. Pierre Judet de la Combe propose une mise en contexte remarquable des Atrides d'Ariane Mnouchkine (1989-1993). Sa contribution mérite une attention particulière: se refusant au témoignage auquel l'autorisait sa participation à «l'aventure », ce dernier a préféré concentrer la réflexion sur la place de l'interprétation d'Ariane Mnouchkine dans une histoire de la dramaturgie européenne moderne. Deux événements fondateurs ont orienté la tradition théâtrale française de représentation de l'Agamemnon: l'entreprise universitaire du Groupe de Théâtre Antique de la Sorbonne et la représentation de l'Orestie par Jean-Louis Barrault à Bordeaux, en 1955. Les courants représentés par Paul Claudel, Antonin Artaud, Bertold Brecht, qui ont tous contribué à produire une interprétation théologique et politique de la pièce, dessinent un «modèle intellectuel » dont les Atrides d'Ariane Mnouchkine ont en partie hérité, même si sa production échappe clairement aux difficultés rencontrées traditionnellement par une interprétation conceptuelle.

6 Le recueil s'achève sur deux ouvertures. La première est consacrée aux réinterprétations scéniques contemporaines $d u$ rôle énigmatique, souvent embarrassant, de la prophétesse Cassandre. La seconde, comme dans le recueil consacré à Médée, clôt l'ouvrage sur un catalogue des représentations d'Agamemnon recensées dans le monde entier par l'APGRD pour la période 458 av. J.-C. à 2004 ap. J.-C. (72 pages de tableaux comportant les noms des metteurs en scène et des détails chronologiques et géographiques). Comme le souligne Amanda Wrigley, auteur du catalogue, la fortune scénique d'Agamemnon ne débuta qu'à la fin du xix ${ }^{\mathrm{e}}$ siècle (1870) alors que Médée était déjà représentée depuis le début du siècle.

7 L'apgrd nous offre, une fois encore, un volume de qualité, conforme à ce que laissaient attendre les précédentes publications. Les contributions ainsi rassemblées balaient un large champ de recherches, ouvrent une multiplicité de voies d'interprétation, tout en respectant une remarquable cohérence (P. Michelakis mentionne, en introduction, les thèmes volontairement écartés ou délaissés). Leurs auteurs ont su allier l'érudition indispensable à toute recherche en matière de réception, la culture et la finesse des interprétations historiques et politiques. L'Agamemnon, les Agamemnon, prennent sens. L'ouvrage donne au lecteur le désir d'aller au cinéma, au théâtre, à l'opéra goûter anciennes et nouvelles mises en scène, ou, tout simplement, riche de tous ces éclairages, de relire les textes d'Eschyle, de Sénèque et de leurs successeurs. 


\section{AUTEURS}

\section{MARIE-HÉLÈNE GARELLI}

Université de Toulouse II-Le Mirail

garelli@univ-tlse2.fr 